\title{
Glottic Cancer cM0 TNM Finding v7
}

National Cancer Institute

\section{Source}

National Cancer Institute. Glottic Cancer CMO TNM Finding v7. NCI Thesaurus. Code C89075.

Glottic cancer without evidence of distant metastasis. (from AJCC 7th Ed.) 